\title{
Poverty in Tunisia: A Fuzzy Measurement Approach
}

\author{
Besma Belhadj $^{a}$ and Mohamed Salah Matoussi ${ }^{\mathrm{b}}$
}

JEL-Classification: P46, I32, D81.

Keywords: fuzzy sets, poverty, poverty line, membership function, information function.

\section{Introduction}

One of the most intractable problems facing researchers and policy makers in the areas of social security and public policy is the identification of "the poor" and the measurement of the extent of poverty. A number of different approaches to the derivation of a poverty line and the measurement of poverty have been proposed and applied (see e.g. Foster, Greer and Thorbecke, 1984; Sen, 1976).

The question of how to determine and set a living condition threshold below which a person or family can be considered poor is very often the source of considerable debate, and the theoretical and empirical literature has proposed ways to avoid ad-hoc approaches. Although the proposals are sophisticated, the action of deriving such thresholds is arbitrary. For example, the use of incomes or expenditure indicators of welfare requires the determination of poverty lines which are supposed to measure deprivation in an absolute sense. Most of the methods designed for the analysis of poverty are based only upon a monetary variable and they dichotomize the population into "poor" and "non-poor" by means of the so called poverty line.

Nowadays many authors recognize that poverty is a complex phenomenon that cannot be reduced solely to the monetary dimension. This leads to the need for a multidimensional approach that consists in extending the analysis to a variety of non-monetary indicators of living conditions (see e.g. Bourguignon and Chakravarty, 2003; Kakwani and Silber, 2008). The multi-dimensional aspect "capability approach" proposes to go beyond the arbitrariness of the monetary approach to poverty measurement. Multi-dimensionality involves both

a Higher Institute of Management, University of Tunisia. The researchers wish to thank the anonymous reviewers for their comments and reviews including all the important points raised.

b University of Economics, University of Tunisia. 
monetary and diverse non-monetary aspects - the former as the situation, either absolute or relative to the average standard, of low income, and the latter as a lack of access to other resources, facilities, social interactions and even individual attributes determining life-style.

By contrast, however, little attention has been devoted to the rigid poor/nonpoor dichotomy, with the consequence that most of the literature on poverty measurement continues to be based on the use of poverty thresholds. Yet it is undisputable that such a clear-cut division causes a loss of information and removes the nuances that exist between the two extremes of substantial welfare on the one hand and distinct material hardship on the other. In other words, poverty should be considered as a matter of degree rather than as an attribute that is simply present or absent for individuals in the population. Fuzzy set theory has been applied to the analysis of unidimensional poverty in SHORRoKs and Subramanian (1994), and Schaich and Munnich (1996). An early attempt to incorporate this concept at a methodological level, and in a multidimensional framework, was made by Cerioli and Zani (1990) and Dagum, Gambassi and Lemmi (2002) who drew inspiration from the theory of Fuzzy Sets initiated by ZADEH (1965). Cerioli and Zani's original proposal was later developed by CHELI and Lemmi (1995) giving origin to the so called Totally Fuzzy and Relative (TFR) approach. Both methods have been applied by a number of authors subsequently, with a preference for the TFR version (for instance, Chiappero Martinetti, 2000). From this point on, the methodological implementation of this approach has developed in two directions, with somewhat different emphasis despite their common orientation and framework. The first of these is typified by the contributions of Betti, Cheli and Cambini (2004), focusing more on the time dimension, in particular making use of the tool of transition matrices. The second, with the contributions of BeTti and Verma (2008), has focused more on capturing the multi-dimensional aspects, developing the concepts of "manifest" and "latent" deprivation to reflect the intersection and union of different dimensions. On the basis of Human Development Index critics, Berenger and VerdierChouchane (2007) treated two different multidimensional analyses of poverty, the Totally Fuzzy Analysis and the Factorial Analysis of Correspondences.

Admittedly the fuzzy approach was specially applied to multidimensional poverty measure. However for the multidimensional approach, the difficulty is to determine the variety of non-monetary indicators of living conditions in the most objective manner and least ideological precise. Such social practice can indeed be regarded as a basic right for reasons returning less to the existence material conditions than to the methods of social integration, i.e. of adhesion to the social standards of behaviour, for example the possession or not of a television or a car. 
Hence, one puts at the same level the deprivation of television and the deprivation of care. Actually the poor will choose to reduce their expenditure of health rather than to be deprived of television.

To avoid any confusion caused by the multidimensional aspect of poverty measurement, a fuzzy poverty index by taking account only of the monetary aspect is proposed.

Measurement of poverty may be sensitive to how the poor are identified. To dichotomize the population into "poor" and "non-poor" groups, each person $i$ is assigned the equivalised income $Y_{i}$ of the person's household. Persons with equivalised income ${ }^{1}$ below a certain threshold or poverty line (such as $50 \%$ of the median equivalised income) are considered to be poor, and the others as nonpoor. One cannot decide on a poverty line which achieves unanimity. Through their use of $0 / 1$ indicators, targeting errors usually used in the literature tend to differentiate drastically between the poor and the non-poor, in particular between those in similar circumstances but who just happen to lie on opposite sides of a poverty line. The targeting efficiency for the poor is typically analysed by building a confidence interval for the poverty line. On the basis of this interval first, three fuzzy subsets are defined and second their membership functions are formulated. Then, a resulting membership function (m.f.) is selected which satisfies all properties of a poverty measurement and is considered as the individual index of deprivation of each unit. A fuzzy information function is used to propose, finally, a fuzzy collective one-dimensional poverty index based only upon a monetary variable.

The information function suggested resembles Watt's poverty index that can be connected, in a direct or indirect way, to certain indices of inequality (MAssoumi, 1993).

The methodology proposed here is illustrated by the Tunisian case. The household survey data conducted by the Tunisian Institute of Statistics (INS) in $1990^{2}$ and involving 7734 households from different regions is used. Unfortunately the household survey does not provide direct information on prices. Instead, it gives detailed information on expenditures, including consumption of own products, and quantities so that local prices can be estimated. Half of the sampled

1 Equivalised income is defined as the net disposable total household income divided by equivalised household size, which takes into account variations in household size and composition. For numerical applications in this paper, we have used the elasticity size.

2 In Tunisia the data are not available what prevents us from carrying out an application with recent data. 
households were included in another survey from which we can get information about goods.

The following sections are devoted to the presentation of the different steps needed for the determination of the fuzzy unidimensional poverty index. Sect. 2 solves the problem of uncertainty of the poverty line. Sect. 3 discusses the methodology of construction of a poverty fuzzy unidimensional index. Sect. 4 illustrates the use of this index utilizing household data from Tunisian for the "ruralurban", spatial comparison, a comparison by activity of the household chief and a comparison by educational level. Sect. 5 concludes.

\section{Uncertainty of the Poverty Line}

Poverty analysis is based on the determination of poverty lines from which one then computes poverty indices such as the head count ratio or more sophisticated ones (see e.g. Zheng, 1997). These indexes can then be used by economists and policy makers for temporal or spatial comparisons in a relatively easy manner. The determination of the poverty line is an important and an uncertain issue, in this section the estimation of the confidence interval is proposed.

We suppose that the poverty line belongs to the interval $\left[z_{\min }-z_{\max }\right]$ where $z_{\min }$ represents the lower poverty line and $z_{\max }$ the upper poverty line. The determination of the lower and upper limits is a delicate step because it is not independent of the socio-economic context in which the individual is established and must take into account of the particular characteristics of the choice of deprivation indicator.

In this paper, we consider the general approach for the assessment of poverty lines adopted by RavalLION and Bidani (1994). This approach consists of determining first the minimum income, to satisfy basic food needs, and second estimating the minimum income to satisfy non food needs. These minimum incomes constitute respectively the food and non food poverty lines. Basic food needs are computed on a regional basis depending on the local food consumer behaviour so that the typical consumption basket ensures a minimal calorific intake as determined by nutritionists. Then, this basket is evaluated using local prices so that the food poverty line can be calculated.

The natural approach is to construct a consumer's basket of non food goods associated to a poor household and then calculate its value by means of local prices. There are however two serious impediments to this approach. The first one is due to the fact that usually one doesn't have data on non-food products and the second is that it is almost impossible to elaborate a homogenous measure 
for the quantities of non food products and deduce representative unit values. We therefore choose to approximate the non food budget share of the poverty line by looking at the behaviour of the household with income equal to the food poverty line. The share, they are ready to sacrifice in order to satisfy their basic needs on non food products, will serve to estimate the non food part of the poverty line.

The valuation of the non food component is carried out by using a method presented in RaVALLION (1994). This approach, based on the intuitive argument that the definition of basic non food needs, requires the valuation to the willingness to give up a necessary food product in order to purchase the required item. Ravallion estimates the value of the food component by an AIDS class of functions:

$$
\omega_{i j}=\alpha_{j}^{0}+\beta_{j} \log \left(\frac{Y_{i j}}{z_{j}^{f} n_{i j}^{\theta}}\right)+\sum_{k} \delta_{j}^{k} d_{i j}^{k}+\varepsilon_{i j}
$$

where $\omega_{i j}$ is the food share of household $i$ belonging to the region and/or area $j$, $Y_{i j}$ is its total per capita expenditure, $z_{j}^{f}$ is the already established food poverty line for area $j, n_{i j}^{\theta}$ is the equivalent size of household $i$ belonging to the region and/ or area $j, \theta$ is elasticity size ${ }^{3}, d_{i j}^{k}$ are socioeconomic variables such as the age of household head, the number of children, the number of working women, etc..., and $\varepsilon_{i j}$ is a disturbance term. A quadratic term in $\log \left(y_{i j} / z_{j}^{f}\right)$ can be added to improve the fit (see Ravallion and Bidani, 1994). The value of

$$
\alpha_{j}=\alpha_{j}^{0}+\sum_{k} \delta_{j}^{k} \bar{d}_{j}^{k}
$$

estimates the expected non food shares of households with per capita expenditure that reaches the food poverty line, i.e. $Y_{i j}=z_{j}^{f}$. The evaluation of $\bar{d}_{j}^{k}$ is made by means of the sub sample with per capita expenditure around the poverty line. The poverty line is then given by $z_{\min j}=z_{j}^{f}+z_{j}^{n f}=z_{j}^{f}+(1-\alpha) z_{j}^{f}=\left(2-\alpha_{j}\right) z_{j}^{f}$ where $z_{j}^{n f}$ is the non-food poverty line for area $j$, and includes de minimum expenditure to satisfy basic food and non food needs. This is actually the socalled lower poverty line and one we can also compute a so-called upper poverty line which then leads to an interval of possible poverty lines.

3 Elasticity size corresponds to the elasticity of equivalence scale compared to the size of household. 
The upper poverty line $z_{\max }$ corresponds to the level of the total expenditure per capita necessary to make it possible the households to satisfy their basic food needs without sacrifice. This poverty line, which can be obtained only by iteration, enables us to estimate the maximum expenditure of non-food which corresponds to the food poverty line ${ }^{4}$.

\section{Methodology of Construction of the Poverty Fuzzy Index}

It is useful to clarify the concept of treating poverty (or more generally, various forms of deprivation) as a degree replacing the conventional classification of the population into a simple dichotomy. Basically, all individuals in a population are subject to poverty, but to varying degrees. We say that each individual has a certain propensity to be poor, the population covering the whole range $[0,1]$. The conventional approach given in Figure 1, is a special case where the population is dichotomised as $\{0,1\}$. Those with an income below a certain threshold are deemed to be poor (i.e. are all assigned a constant propensity $=1$ ); others with income equal or above that threshold are deemed to be non-poor (i.e. are all assigned a constant propensity $=0$ ).

As to the fuzzy sets, the basic idea is as follows. Given a set $X$ of elements $x \in X$, any fuzzy subset $A$ of $X$ is defined as: $A=\left\{\mathrm{x}, \mu_{A}(x)\right\}$ where $\mu_{A}(x): X \rightarrow[0,1]$ is called the $m . f$. in the fuzzy subset $A$. The value $\mu_{A}(x)$ indicates the degree of membership of $x$ in $A$. Thus $\mu_{A}(x)=0$ means that $x$ does not belong at all to $A$, whereas $\mu(x)=1$ means that $x$ belongs to $A$ completely. On the other hand, when $0<\mu_{A}(x)<1$ then $x$ partially belongs to $A$ and its degree of membership of $A$ increases in proportion to the proximity of $\mu_{A}(x)$ to 1 (Kaufmann and Gupta, 1991).

4 One proceeds by iteration until finding the level of total expenditure per capita such as:

$$
z_{j}^{A}=E\left(\alpha \frac{Y_{j}}{n_{j}^{\theta}}=z_{\max j}\right) z_{\max j} .
$$


Figure 1: The Conventional Poor/Non-Poor Dichotomy

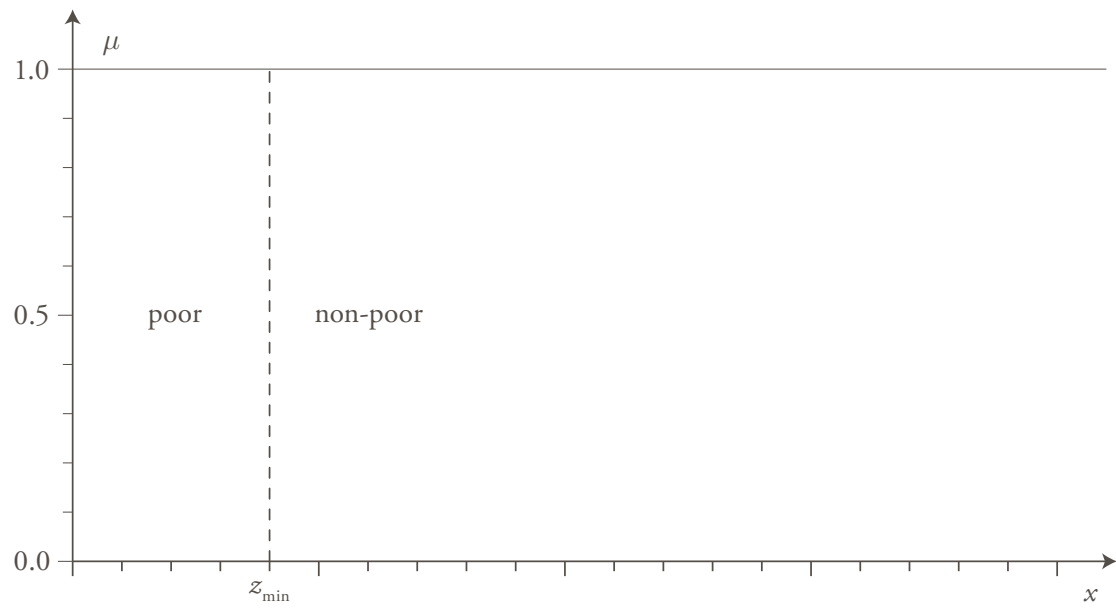

\subsection{Internal Configuration by Fuzzy Logic}

In this section a sort of step-by-step procedure is described. The first step that must be undertaken requires identifying the relevant evaluative domains and specifying the corresponding fuzzy sets appropriately. As RAGIN (2000) points out, specifying fuzzy sets is different and more complex than specifying variables. In this step, first the "poverty line" input variable is considered and three levels of this variable are constructed: a low level, a medium level and a high level. Consequently, three fuzzy subsets for the poverty line are deduced as shown in Figure 2: Weak, Medium and High. Second, "poverty" is defined as a output variable. Three fuzzy subsets are proposed as shown in Figure 3: "Strong Privation" $(S P)$, "Medium Privation" $(M P)$ and "Weak Privation" (WP).

The logic of these scales of privation intensity is as follows: in the case of the monetary variables such as the income or the expenditure, one is confronted in general with situations where the living conditions improve with an increase of the indicator.

Fuzzy methodology translates these ordinal ranks into fuzzy membership scores or degrees that are capable of reflecting the content of the ordinal categories in line with our conceptual understanding of the phenomenon that we want to describe.

This leads us to the second step, i.e. how to assign membership degrees or scores and to calibrate appropriate membership functions, in section 3.2. 
Figure 2: Confidence Interval of the Poverty Line

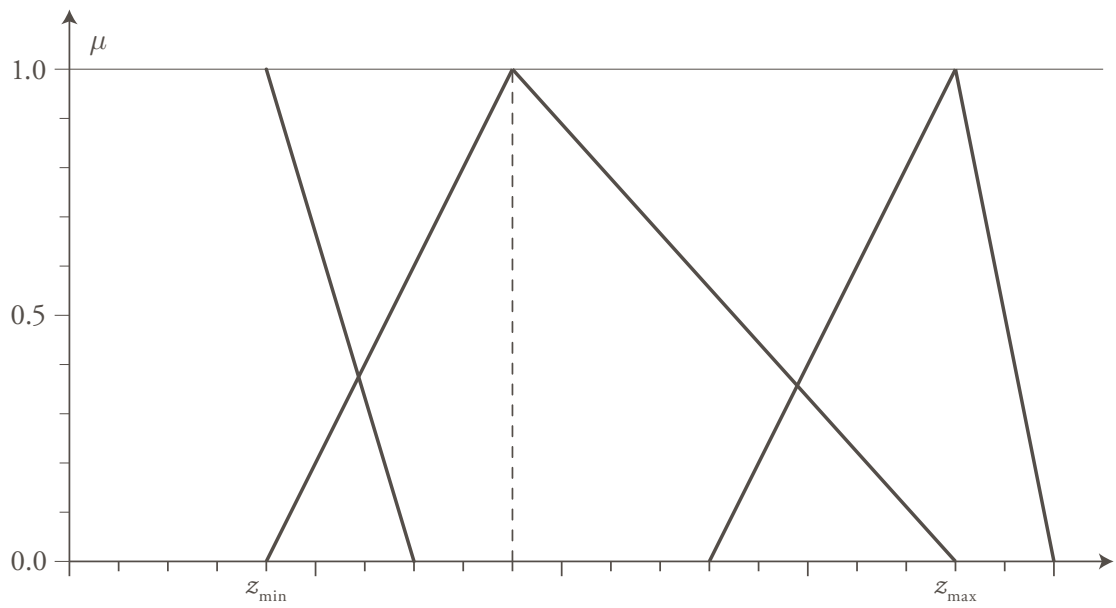

- "Strong privation" corresponds to a poverty line lower or equal to its minimal value $z \leq z_{\min }$ and, consequently, the deprivation is a very relevant poverty-indicator.

- "Medium privation" corresponds to a poverty line belonging to the selected interval $\left[z_{\min }-z_{\max }\right]$.

- "Weak privation" corresponds to a poverty line beyond its maximum value $z \geq z_{\max }$ and the deprivation cannot be considered alone as a reliable index of poverty.

Figure 3: The Poverty Modalities

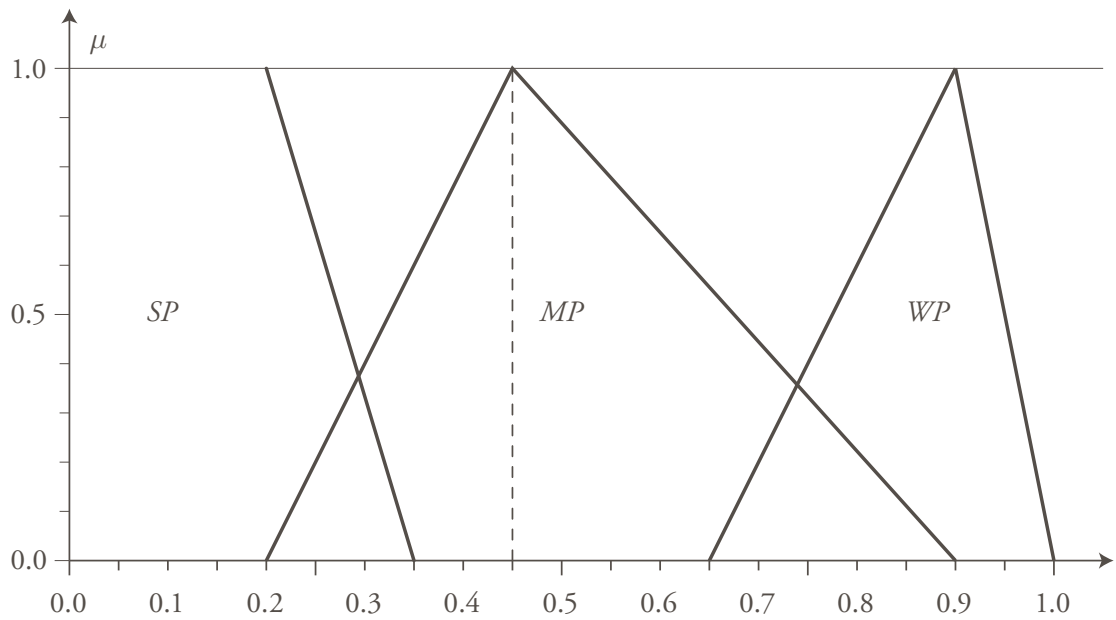


Again, this step is neither automatic nor univocal as it would be in the case of an ordinal scale.

In the third step a method of "Composition of rules" is proposed to transform fuzzy information into representative values, see section 3.3.

\subsection{Constructing Membership Functions}

Different methods can be adopted for constructing membership functions (CHIappero Martinett, 2006). They can be chosen arbitrarily by the investigator, according to her or his common sense and experience, or the value judgements underlying the theoretical concept that she or he wishes to describe. For instance, a simple decreasing or increasing linear membership function can be adequate in order to depict variables or concepts distributed along a linear continuum between 0 and 1 (inclusive), where any value is proportional to its distance in the value axis. Triangular or Trapezoidal-shaped membership functions make it possible to preserve linearity and at the same time to incorporate minimum and/or maximum thresholds: this can be helpful in adapting variables to different realities or circumstances. Nonlinear membership functions - such as sigmoid or logistic curves - can make it possible to "fine-tune" the representation and require identifying not only the two extreme 0 and 1 membership values, but also the flex or crossover point associated to a membership degree equal to 0.5 , according to criteria established by the investigator. Other nonlinear functions, such as Gaussian or exponential curves or irregularly shaped functions can be equally applied.

What seems to emerge clearly is that whatever criteria one adopts to construct membership functions, they should be able to convey the semantic properties of the underlying concept; the closer the membership function maps the nature and behaviour of our conceptual phenomenon, the better it will reflect the real word that we want to describe. We suggest defining the function of membership of each fuzzy subset as a linear function of the income takes with values in interval $[0,1]$. We choose, consequently, the Triangular membership function (TMF) to preserve this linearity and at the same time to distinguish between the three levels of poverty (SP, MP and WP).

To measure the individual poverty, we proceed as follows: First, the $m . f$. of each fuzzy subset (SP, MP and WP) is formulated. Second, a resulting $m$.f. of the poor that satisfies the properties of a measurement of poverty is selected. Third, a unidimensional poverty fuzzy index is proposed using an Information Theory approach. 
Formally, the $m . f$. of each fuzzy subset is as follows:

$$
\begin{aligned}
& \mu_{S P}(i)=\left\{\begin{array}{lc}
1 & 0<x_{i}<z_{\min } \\
\frac{-1}{P_{1}-z_{\min }} x_{i}+\frac{P_{1}}{P_{1}-z_{\min }} & z_{\min } \leq x_{i}<P_{1} \\
0 & x_{i} \geq P_{1}
\end{array}\right. \\
& \mu_{M P}(i)= \begin{cases}0 & x_{i} \leq z_{\min } \\
\frac{2}{z_{\max }-z_{\min }} x_{i}-2 \frac{z_{\text {min }}}{z_{\text {max }}-z_{\min }} & z_{\min } \leq x_{i}<z_{B} \\
\frac{-2}{z_{\max }-z_{\min }} x_{i}+\frac{2 z_{\max }}{z_{\text {max }}-z_{\min }} & z_{B} \leq x_{i}<z_{\max } \\
0 & x_{i} \geq z_{\max }\end{cases} \\
& \mu_{W P}(i)=\left\{\begin{array}{lc}
0 & x_{i} \leq P_{2} \\
\frac{1}{z_{\max }-P_{2}} x_{i}-\frac{P_{2}}{z_{\max }-P_{2}} & P_{2} \leq x_{i}<z_{\max } \\
\frac{-1}{P_{3}-z_{\max }} x_{i}+\frac{P_{3}}{P_{3}-z_{\max }} & z_{\max } \leq x_{i} \leq P_{3} \\
0 & x_{i} \geq P_{3}
\end{array}\right.
\end{aligned}
$$

$x_{\mathrm{i}}$ being the income or the expenditure of the household $i, P_{1}$ is a certain value of the income or expenditure higher than $z_{\min }, P_{2}$ is a certain value of the income or expenditure lower than $z_{\max }$ and $z_{B}$, as shown in Figure 4 , is the barycentre of the interval $\left[z_{\min }-z_{\max }\right]$.

It is worth underlining that between the two thresholds $z_{\min }$ and $z_{\max }$, the $m . f$. takes its values in interval $[0,1]$. A $m . f$. chosen as the degree of poverty of household $i$ must be a non-increasing function for variables such as the income or the expenditure, for which an increase translates an improvement in the situation of welfare. 
Figure 4: The Values of Poverty Line

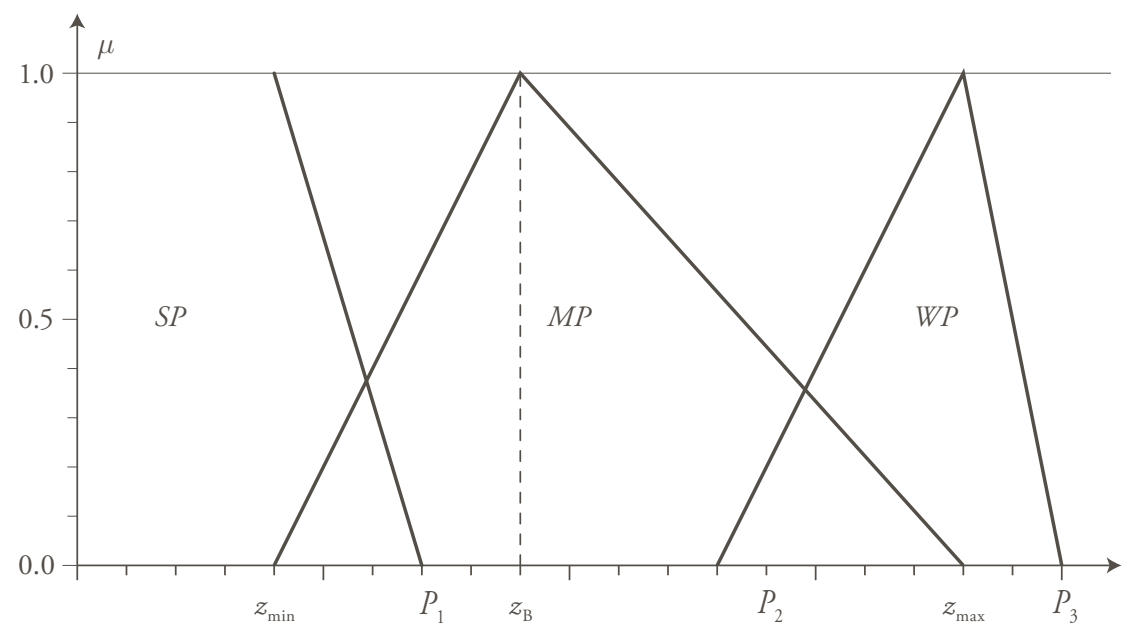

$\mu_{S P}(i), \mu_{M P}(i)$ and $\mu_{W P}(i)$ respectively indicate the membership function of the household $i$ of the fuzzy subsets "SP", "MP" and "WP".

Consequently, we propose to define the resulting $m . f$. (see Figure 5) in the following way:

$$
\mu_{Q}(i)= \begin{cases}1 & 0<x_{i}<z_{\text {min }} \\ \frac{-x_{i}}{z_{\text {max }}-z_{\text {min }}}+\frac{z_{\text {max }}}{z_{\text {max }}-z_{\text {min }}} & z_{\min } \leq x_{i}<z_{\max } \\ 0 & x_{i} \geq z_{\max }\end{cases}
$$

Equation (5) gives the fuzzy individual poverty index of a household $i$. The $m$. $f$. of Equation (5) takes its values in interval [0,1]. It represents the membership degree of a household $i$ to fuzzy subset of poor. The $m . f$. is a non-increasing function. It satisfies all properties of a poverty measurement. Indeed, an increase in the income or expenditure involves a reduction in the degree of individual poverty and an increase in the living conditions. 
Figure 5: The Resulting Membership Function

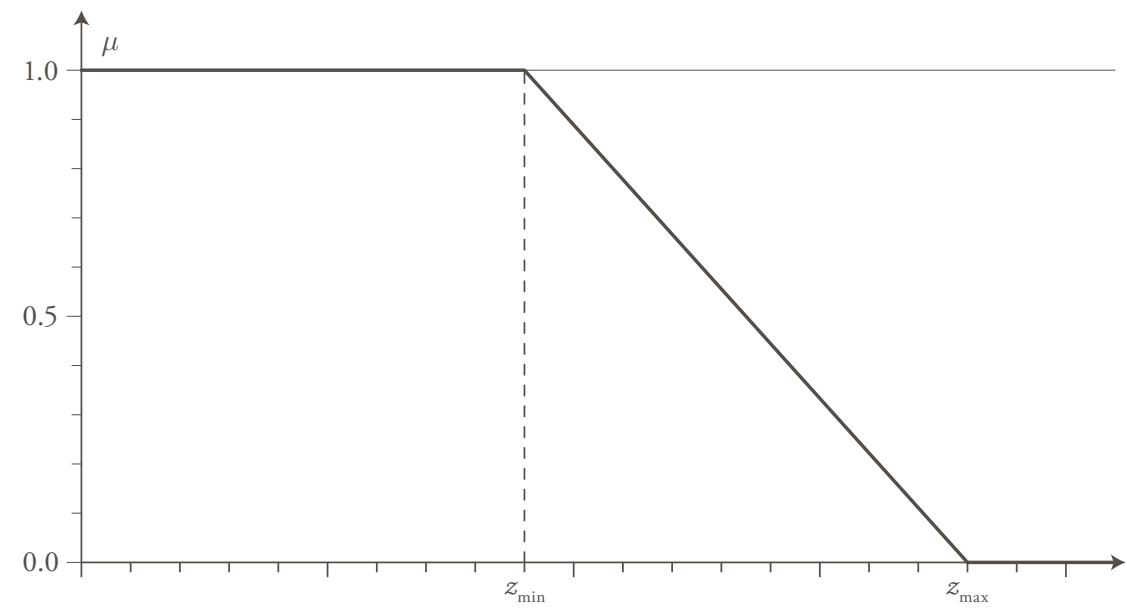

\subsection{Fuzzy Unidimensional Poverty Index}

Referred to the overall population, we propose to use an Information Theory approach to design a fuzzy collective index.

A brief description of the Information Theory (IT) approach is as follows: Information theory is based on probability theory and statistics (TheIL, 1967). The most important quantities of information are entropy, the information in a random variable, and mutual information, the amount on information in common between two random variables. The former quantity indicates how easily message data can be compressed while the latter can be used to find the communication rate across a channel.

We propose the following as the underlying fuzzy collective poverty index:

$$
I(\mu, z)=\sum_{i \in Q} q_{i} \log \left(\frac{q_{i}}{p_{i}}\right),
$$

where a prior distribution is given by the distribution of individuals:

$$
p_{i}=1 / n, \forall i ; \sum_{i} p_{i}=1,
$$


and a posterior distribution is given by the share of membership of a household $i$ to the fuzzy subset of the poor:

$$
q_{i}=\frac{\mu_{Q}(i)}{\sum_{i} \mu_{Q}(i)}, i=1 \ldots n, \sum_{i} q_{i}=1 .
$$

Equation (6) is a way of comparing two distributions: a prior probability distribution $p_{1}, \ldots, p_{n}$, and a posterior probability distribution $q_{1}, \ldots, q_{n}$. The choice of logarithmic base in the preceding formulae determines the unit of information entropy that is used.

$I\left(\right.$.) is in the majority of cases positive and takes a zero value only when $p_{i}=q_{i}$, i.e. when the new message leaves the probabilities completely unchanged.

$I\left(\right.$.) is an increasing average of the degree of poverty of household $i, \mu_{Q}(i)$, i.e. a deterioration of the living conditions of a household belonging to the fuzzy subset of poor causes a increase of the poverty general index.

We notice that, like Watt's poverty index, (6) is an information function. It is fuzzy relative entropy. Watt's poverty index ${ }^{5}$ is one of the major axiomatically sound poverty indexes, exhibits additional interesting properties.

The index $I($.) satisfies the focus, monotonicity, transfer and transfer sensitivity axioms. It is also continuous, subgroup consistent and even decomposable.

\section{Empirical Analysis}

In this section, the methodology of section 3 is applied not to compare different regions in Tunisia, but also to compare poverty in rural versus urban regions, according to the activity of the household chief, and according to the educational level.

5 The Watt's index is expressed as follows:

$$
W=\frac{1}{n} \sum_{i \in Z(x)} \log \left(\frac{z}{x_{i}}\right)
$$

where $z$ is the poverty line relating to the distribution $x$ and $Z(x)$ is the set of poor. 


\subsection{Estimation of the Interval}

As said in the introduction, the data we are using from the household survey data conducted by the INS in 1990 involving 7734 households. The sampling scheme and the results of the survey are explained in INS (1990). The survey also provides the demographic characteristics of households. In order to take into account the different geographical and socioeconomic characteristics of the regions in Tunisia, the country is divided in 5 different homogenous regions (see the Appendix), three of which are urban areas.

Three indicators of deprivation are retained:

- The economic area: Greater Tunis, urban littoral, rural littoral, urban interior, and rural interior.

- The activity of the household chief: inactive, farm labourer, farmer, independent non-agricultural, owner and tallies, others.

- The educational level of the household chief: illiterate, primary, secondary 1st cycle, secondary 2-er cycles, university.

In the following tables, the interval of the poverty line expressed in Tunisian dinars and the poverty fuzzy index per indicator are calculated.

The estimated food poverty lines for the 5 regions of Tunisia are presented in the first column of Table 1. "We observe that in 1990 for the "Greater Tunis" region, for example, any household whose annual expenditure is lower than 280DT is considered poor and its degree of membership to the fuzzy subset "Strong Privation" is very high. On the other hand, any household whose annual total expenditure exceeds 339DT is judged to be non-poor. The degree of membership of the fuzzy subset "Weak Privation" is high.

The poverty lines estimated are lower in the poorest regions: Interior Urban, Interior Rural and Littoral Rural.

However, what attracts our attention is the difference between the urban and rural lines. The urban/rural ratio in the littoral region is equal to 1.47 . On the other hand, the ratio urban/rural in the Interior is equal to 1.25. Indeed, the urban littoral has seen a rapid economic development compared to the interior

6 We grouped Greater Tunis and Littoral urban together to compute the consumer's basket because the respective samples were relatively small. We however considered different unit values and therefore poverty lines for the two regions. 
Table 1: Interval of the Poverty Line According to Various Economic Areas (1990)

\begin{tabular}{lcc}
\hline Economic area & $z_{j}^{f}$ & {$\left[z_{\min }-z_{\max }\right]$} \\
\hline Greater Tunis & 191 & {$[280-339]$} \\
Urban Littoral & 180 & {$[252-313]$} \\
Urban Interior & 145 & {$[212-292]$} \\
Rural Littoral & 126 & {$[172-219]$} \\
Rural Interior & 122 & {$[169-229]$} \\
\hline
\end{tabular}

Table 2: Average Fuzzy Poverty by Area (1990)

\begin{tabular}{lccc}
\hline Economic area & $\omega_{R}$ & $I$ & $\mu_{Q}$ \\
\hline Greater Tunis & 0.25 & 0.061 & 0.33 \\
Urban Littoral & 0.15 & 0.048 & 0.27 \\
Urban Interior & 0.20 & 0.123 & 0.72 \\
Rural Littoral & 0.22 & 0.107 & 0.56 \\
Rural Interior & 0.18 & 0.124 & 0.74 \\
Fuzzy poverty & & 0.094 & \\
\hline
\end{tabular}

$\omega_{R}$ represent the relative frequency of each area.

which has led to an increase in living costs and which explains why the urban/ rural difference in the littoral region should be greater than the one in the interior region.

\subsection{Regional Fuzzy Poverty}

When inspecting the second column of Table 2, one first notices that poverty in Tunisia during the year 1990 is a phenomenon that affects the Interior Rural and Interior Urban more severely. These areas present a deprivation stronger than the other areas, the fuzzy measurement of poverty in 1990 for these two areas is about 0.25 and 0.123 .

The results show that, in 1990, average fuzzy poverty was about 0.094 and that the areas "Littoral Urban" and "Greater Tunis" present on average living condition different from the others and better than the national average. 


\subsection{Fuzzy Poverty by Activity of the Household Chief}

It's clear from the second column of Table 3 that, in the Tunisian average, farm labourers and non-agricultural workers are touched by poverty. Their contribution to the fuzzy subset "Medium" is respectively about $68 \%$ and $73 \%$. By taking into account these results, we can note that any structural Tunisian socioeconomic policy to reduce poverty must include a reform aiming at helping this socio-professional category.

By inspecting fourth column of Table 3, we note that estimated poverty lines are lower for the farm labourers and non-agricultural workers. The employer/ non-agricultural ratio is equal to 2.14. Consequently, the education level is a reduction factor of the monetary poverty.

Table 3: Average Fuzzy Poverty According to Profession (1990)

\begin{tabular}{lcccc}
\hline Profession & $\omega_{F}$ & $I$ & $\mu_{Q}$ & {$\left[z_{\min }-z_{\max }\right]$} \\
\hline Inactive & 0.094 & 0.051 & 0.38 & {$[167-245]$} \\
Farm labourers & 0.180 & 0.103 & 0.68 & {$[156-228]$} \\
Farmers & 0.059 & 0.082 & 0.47 & {$[178-226]$} \\
Nonagricultural worker & 0.457 & 0.121 & 0.73 & {$[152-219]$} \\
Independent agricultural & 0.118 & 0.075 & 0.39 & {$[179-256]$} \\
Employer and Managers & 0.015 & 0.005 & 0.18 & {$[325-425]$} \\
Others & 0.077 & 0.032 & 0.25 & {$[185-201]$} \\
Fuzzy poverty & & 0.095 & & \\
\hline
\end{tabular}

$\omega_{F}$ represent the relative frequency of each activity

\subsection{Fuzzy Poverty by Educational Level}

It is clear from the fourth column of Table 4 that, the poverty lines estimated are lower for the illiterates. The difference between the poverty lines estimated of the primary education and secondary 1st cycle is weak. The secondary 1st cycle /primary education ratio is equal to 1.03 . The results showed (Tables 2-4) that, in 1990, poverty in Tunisia affected more severely the regions: "Interior Rural", "Littoral Rural" and "Interior Urban" this contradicted the finding of governmental institutions. These regions must bring all the attention, if ever a reduction program of poverty based on a regional targeting is setup. 
Table 4: Average Fuzzy Poverty According to the Educational Level (1990)

\begin{tabular}{lcccc}
\hline Educational level & $\omega_{i}$ & $I$ & $\mu_{Q}$ & {$\left[z_{\min }-z_{\max }\right]$} \\
\hline Illiterate & 0.464 & 0.125 & 0.78 & {$[152-199]$} \\
Primary education & 0.344 & 0.079 & 0.44 & {$[179-225]$} \\
Secondary 1st cycle & 0.100 & 0.039 & 0.35 & {$[185-292]$} \\
Secondary 2-er cycles & 0.071 & 0.026 & 0.19 & {$[295-325]$} \\
Academic & 0.021 & 0.007 & 0.11 & {$[325-456]$} \\
Fuzzy poverty & 0.091 & & & \\
\hline
\end{tabular}

$\omega_{I}$ represent the relative frequency of each educational level

According to the activity of the household chief, poverty is more significant among the farm labourers and non-agricultural.The intensity of poverty is low at the households whose chief is either top management or middle management, or worker in industry, the trade or the services.

Lastly, if the targeting is made according to the educational level of the household chief, this reveals that the illiterates must hold all the attention. As this fringe of the population occupies the greatest contribution to the measurement of poverty, we can conclude that an intervention of targeting this group can involve one noticeable improvement of the population welfare of the poor.

\section{Conclusion and Research Prospects}

In this paper a new fuzzy poverty index based only on a monetary variable is proposed. This index depends on the definition of a membership function and relative entropy. As an illustration of our methodology we have investigated poverty in Tunisia, paying special attention to urban versus rural comparisons, comparisons according to the activity of the household chief, and comparisons according to the educational level. A fuzzy approach is implemented not only to the determination of the poverty lines but also to compute monetary poverty individual and collective. This approach seems to be very attractive to deal with a problem such as poverty especially with the difficulty of classifying the households in the category of the "poor" or "non poor". By using the fuzzy and the IT approaches, we proposed fuzzy poverty index of each household, the population of the households and the population of the households by attribute. These indices represent the state of poverty and contribute to identify causes of poverty 
structural allowing the development of socio-economic policies aiming at reducing this state.

The results showed that in 1990 poverty in Tunisia was clearly a rural phenomena and this contradicted the finding of governmental institutions. This remains true even if one adopts the fuzzy approach which tends to moderate the differential between rural and urban areas. We also noted that poverty has affected more severely the Interior regions, the farm labourers and non-agricultural and the illiterate people

It is interesting to analyse income poverty dynamics according to a fuzzy approach that unlike conventional methods is consistent with the vague nature of poverty and preserves all the available statistical information. The dynamics of poverty substitutes for the policies of assistance, aiming at increasing the poor incomes of households on a given date, the policies structural acting on the level of the causes of the persistence of the situations of poverty. The measurements of chronic and transient poverties can then be used by economists and policymakers for temporal or spatial comparisons in a relatively easy fashion.

\section{Appendix: Determination of Five Homogenous Regions in Tunisia}

To make use of the characteristics of different regions in Tunisia, we separated the households according to their location with respect to 5 different homogenous regions. Tunisia is traditionally subdivided into three natural regions: North, Center and South. This decomposition is motivated by the geographical characteristics of the country. However, from an economic point of view, it is more appropriate to divide Tunisia into three parts: The Greater Tunis and two homogenous sets namely the Littoral and the Interior. The Greater Tunis area, which involves almost $25 \%$ of the total population, is characterised by very special administrative, social and economic properties. The Tunisian Littoral (Bizerte, Cap-Bon, Sahel, Sfax and Gabes) have known since the independence an economic and social prosperity. This coastal fringe extending from North to South contains, together with the Greater Tunis area, the essential of the tourist, industrial and urban activity of the economy. Despite a certain economic progress, the interior region has several acute social and economic problems which distinguish it from the other two regions. If one compares the per capita expenditure (during 1990), one sees that this subdivision is justified. In addition to this regional decomposition, it is necessary to take into account the rural-urban distinction. We also aggregated the rural part of the Greater Tunis and the littoral. Two reasons support this aggregation. First, the size of the rural Greater Tunis is 
very small, only 167 households and second, the rural of Greater Tunis and those of the rest of the littoral are very similar and can be lumped together to form a homogenous spatial set. This leads us to five homogenous regions, namely the urban Greater Tunis, the urban Littoral, the urban Interior, the rural Littoral and rural Interior.

\section{References}

Berenger, V., and A. Verdier-Chouchane (2007), "Multidimensional Measures of Well-Being: Standard of Living and Quality of Life Across Countries", World Development, 35 (7), pp. 1259-1276.

Betti, G., G. Cheli and R. Cambini (2004), "A Statistical Model for The Dynamics Between Two Fuzzy States: Theory and Application to Poverty Analysis", Metron, 62 (3), pp.391-411.

Bourguignon, F., and S. R. Chakravarty (2003), "The Measurement of Multidimensional Poverty", Journal of Economic Inequality, 1 (1), pp. 25-49.

Cerioli, A., and S. Zani (1990), "A Fuzzy Approach to the Measurement of Poverty", in: C. Dagum and M. Zenga, (eds), Income and Wealth Distribution, Inequality and Poverty, Studies in Contemporary, Economics, Springer Verlag, Berlin, pp. 272-284.

Cheli, B. and A. Lemmi. (1995), "Totally Fuzzy and Relative Approach to the Multidimensional Analysis of Poverty”, Economic Notes, 24, pp. 115-134.

Chiappero Martinetti, E. (2000), "A Multidimensional Assessment of WellBeing Based on Sen's Functioning Approach”, Rivista Internazionale di Scienze Sociali, 108, pp. 207-239.

Chiappero Martinetti, E. (2006), "Capability Approach and Fuzzy Set Theory: Description, Aggregation and Inference Issues", in A. Lemmi and G. Betti (eds), Fuzzy Set Approach to Multidimensional Poverty Measurement, Springer + Business Media, LLC, New-York, pp. 139-153.

Dagum, C., R. Gambassi and A. Lemmi (1992), "New Approaches to the Measurement of Poverty. In Poverty Measurement of Economics in Transition", Polish Statistical Association \& Central Statistical Office, Warsaw.

Foster, J., J. Greer and E. Thorbecke (1984), "A Class of Decomposable Poverty Measures”, Econometrica, 52, pp.761-765.

INS (1990), Enquête sur le budget et la consommation des ménages en Tunisie, Tunisian Institute of Statistics, Ministère du plan, Tunis.

KaKWAni, N., and J. Silber (2008), Quantitative Approaches to Multidimensional Poverty Measurement, Palgrave Macmillan. 
Kaufmann, A., and M. M. Gupta (1991), Introduction to Fuzzy Arithmetic, International Thomson Computer Press.

Massoumi, E. (1993), "A Compendium to Information Theory in Economics and Econometrics", Econometric Reviews, 12 (2), pp. 137-181.

Ragin, C. C. (2000), Fuzzy Set Social Science, The University of Chicago Press, Chicago.

Ravallion, M. (1994), Poverty Comparisons, Fundamentals of Pure and Applied Economics Series, Harwood Academic Press, New York.

Ravallion, M. and B. Bidani (1994), "How Robust is a Poverty Profile?", The Word Bank Economic Review.

Schaich, E., and R. (1996), "Der Fuzzy-Set-Ansatz in der Armutsmessung", Jahrbücher für Nationalökonomie and Statistik, 215, pp. 444-469.

Sen, A. K. (1976), "Poverty: An Ordinal Approach to Measurement", Econometrica, 44, pp. 219-231.

Shorroks, A. F. and S. Subramanian (1994), "Fuzzy Poverty Indices", mimeo, University of Essex.

Theil, H. (1967), Economics and Information Theory, Rand McNally \& Company, Chicago.

Watts, H. W. (1967), "The Iso-Prop Index: An Approach to the Determination of Differential Poverty Income Thresholds", The Journal of Human Resources, 2, pp.3-18.

Zadeh, L. (1965), "Probability Theory and Fuzzy Logic are Complementary rather than Competitive", Technometrics, 37, pp. 271-276.

Zheng, B. (1997), "Aggregate Poverty Measures", Journal of Economic Surveys, 11, pp. 123-162.

\section{SUMMARY}

Although poverty is widely recognised as a multidimensional phenomenon, we still believe that monetary aspect has a fundamental role and therefore deserves a special treatment. For this reason we propose an individual unidimensional measure according to a fuzzy approach that, unlike conventional methods, is consistent with the vague nature of poverty and preserves all the available statistical information. Referred to the overall population, we use an Information Theory approach to design unidimensional fuzzy collective index. The methodology proposed here is illustrated by means of the Tunisia case. 